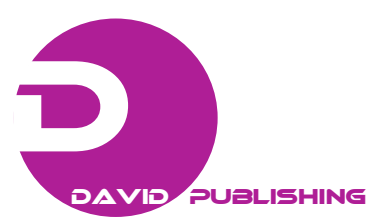

\title{
Combination of Slightly Acidic Electrolyzed Water, Ultrasound and Water Wash to Maximize the Sanitization Effect against Microbial Contamination on Lettuce
}

\author{
Fereidoun Forghani, Myoung-Su Park, Xi-Hong Zhao, Jun Wang, Joong-Hyun Park, Gwang-Hee Kim, Bo-Geum \\ Park and Deog-Hwan Oh* \\ Department of Food Science and Biotechnology and Institute of Bioscience and Biotechnology, Kangwon National University, \\ Chuncheon, Gangwon 200-701, Republic of Korea \\ *Corresponding author's e-mail: deoghwa@kangwon.ac.kr
}

\begin{abstract}
Microbial contamination of fresh produce is still a major concern to the food industry regardless of all recent improvements in food safety technologies. Among the numerous technologies used slightly acidic electrolyzed water (SlAEW) and ultrasound, are both known as environmental friendly technologies. Although, SlAEW still needs more improvement to be used in food sanitization and ultrasound alone does not effectively reduce microbial numbers in food samples. Hence, the aim of this study was to develop an improved hurdle for fresh produce sanitization using combined treatments of SlAEW (pH 5.4, available chlorine concentration $22 \mathrm{mg} / \mathrm{L}$, oxidation reduction potential 544-592 mV) with ultrasound. Lettuce samples were treated with distilled water (DW), SlAEW, SlAEW followed by water wash (SlAEW + WW), SlAEW with ultrasound (SlAEW + US) and SlAEW with ultrasound followed by water wash (SlAEW + US +WW) at room temperature $\left(24 \pm 2{ }^{\circ} \mathrm{C}\right)$, respectively. Following treatments, number of yeasts and molds (YM), total bacteria (TBC) and artificially inoculated Escherichia coli O157:H7 were enumerated. The results showed that ultrasound and water wash alone did not significantly increase the sanitation efficacy of SlAEW. However, treatment of the sample with SlAEW and ultrasound simultaneously, followed by water wash (SlAEW + US + WW) resulted in a great enhancement in microbial reduction. This combined treatment resulted in the reduction of 3.7, 4.1 and 3.8 log CFU/g in YM, TBC and E. coli O157:H7 respectively. Therefore, there is a great chance that this simple hurdle technology can be used in fresh produce as well as other types of food industry to improve the safety of product. Furthermore, addition of mild heat or other treatments should be investigated. This can result in higher numbers of microbial inactivation with cheap, easy to operate and environmental friendly technology.
\end{abstract}

Key words: Slightly acidic electrolyzed water (SlAEW), ultrasound, food sanitization, microbial reduction, lettuce. 